\title{
Vasculature and Bone: Stages of Atherosclerosis come along with Changes in Gene Expression Levels of Calcification Regulators
}

EndoGenelalis

KAGes Schweighofer N. ${ }^{1}$, Aigelsreiter A. ${ }^{2}$, Graf-Rechberger M. ${ }^{2}$, Hacker N. ${ }^{1}$, Kniepeiss D. ${ }^{3}$, Stiegler P. ${ }^{3}$, Trummer O. ${ }^{1}$, Wagner, D. ${ }^{3}$, Pieber T. ${ }^{1}$, Müller H. ${ }^{3}$, Obermayer-Pietsch B. ${ }^{1}$

'Department of Internal Medicine, Divison of Endocrinology and Metabolism , ${ }^{2}$ Institute of Pathology,

${ }^{3}$ Department of Surgery, Division of Transplantation Surgery, Medical University of Graz, Austria

Introduction and aim:

Calcification in the vasculature is one of the leading causes of cardiovascular diseases and mortality outcomes. The present knowledge about expression of calcification regulators in bone compared to vasculature is fragmentary. Therefore, the aim of our study was to investigate changes in the gene expression of calcification regulators (CR) in arterial vessels during different stages of atherosclerosis and to document potential corresponding changes in the bone. Atherosclerotic changes in the vessels were defined by three histological stages of atherosclerosis: $(0)$ no changes, (1) intima thickening or (2) intima calcification. Bone tissue samples were subgrouped accordingly.

\section{Material and methods:}

Gene expression levels of OPG, RANKL, OPN, MGP, BSP-II and RUNX2 were determined in bone, aorta and arteria ilica externa tissue samples of 21 transplant donors. Realtime PCR was performed with predesigned TaqMan assays (Life Technologies) using the LC480 (Roche). Normalisation of the Cp values of CRs was done by division. Beta actin was used as housekeeping gene. The influence of PTH, 25(OH)-Vitamin-D3 and 1,25(OH)2-Vitamin-D3 on the expression of CR was tested in HEK293, HOS, EA.hy926 cells and HUVECs.
Fig. 1: Changes in the expression of CR in arteria iliaca (Al), aorta (AO) and bone (B), in 3 stages of atherosclerosis. The expression (normalised $\mathrm{Cp}$ values) of MGP, OPG and OPN in stage 1 was significantly lower in bone than in vascular tissue. In stage 2, the expression of OPG and OPN decreased in vascular tissue as low as in bone, abolishing the previously seen difference. Stage 0 : Al: $n=3, A o: n=3, B: n=2$; stage 1 : $A$, Ao and $B: n=11$; stage $2: A l: n=8$, Ao and $B: n=7$.

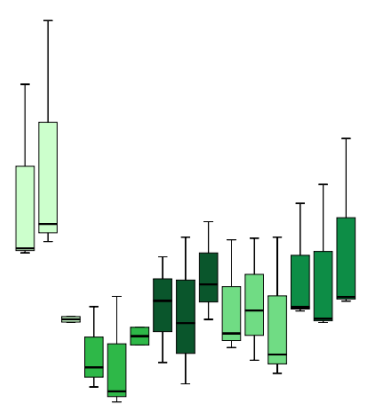

stage 0

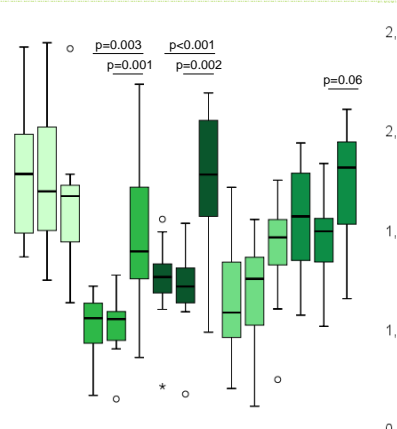

stage 1

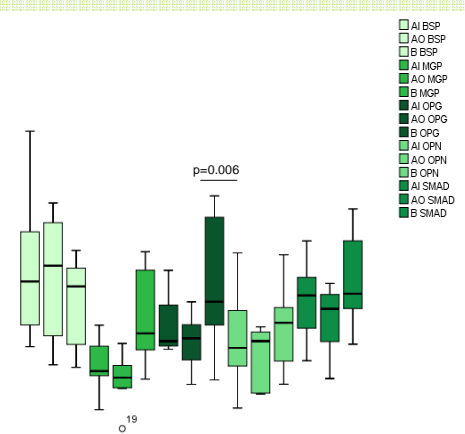

stage 2
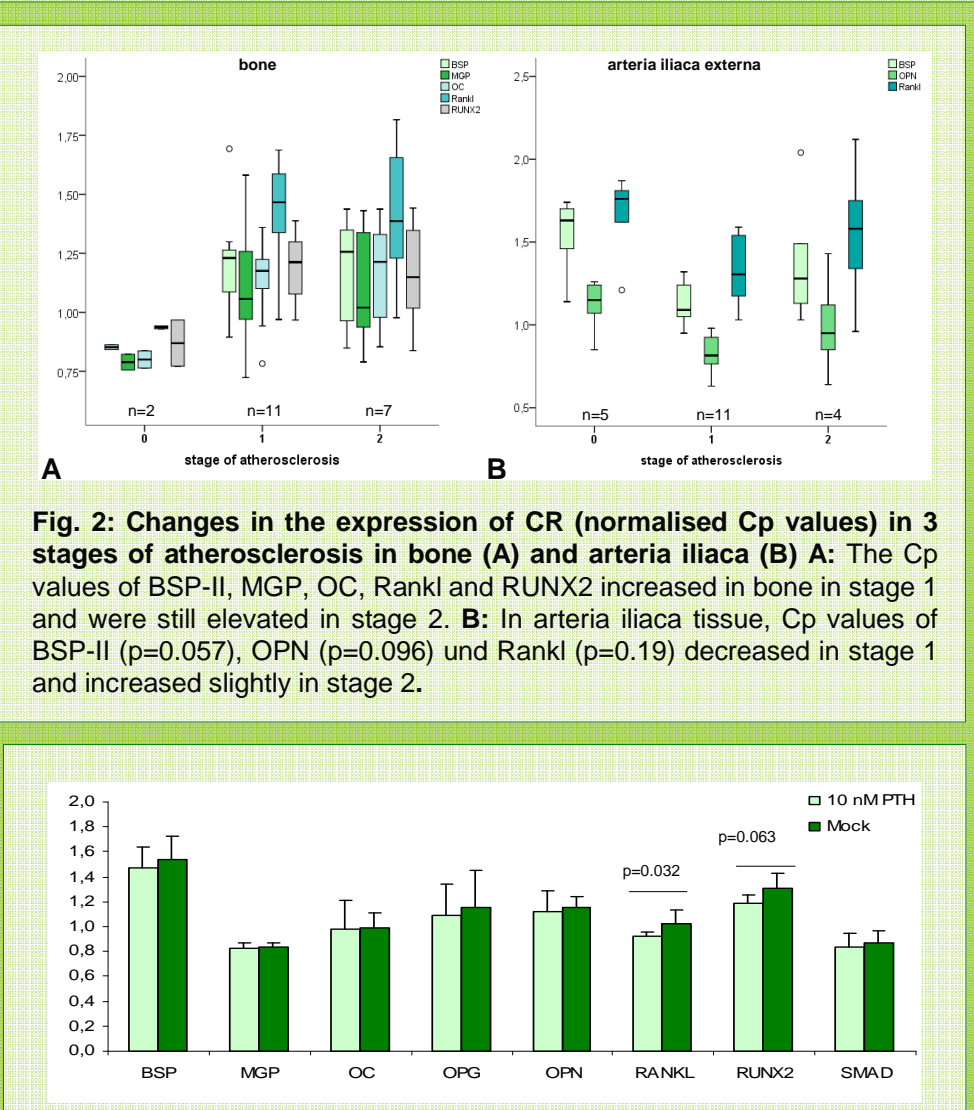

Fig. 4: Changes of expression of CR in HUVECs following addition of $10 \mathrm{nM}$ PTH: The expression of Rankl and RUNX2 (relative Cp values) increased after addition of PTH. Addition of the vitamin D3 metabolites $25 \mathrm{OH}$ - and 1,25(OH)2-Vitamin-D3 did not change the expression of CR. None of the tested metabolites was able to significantly change the expression of CR in HEK293, EA.hy926 and HOS cells.

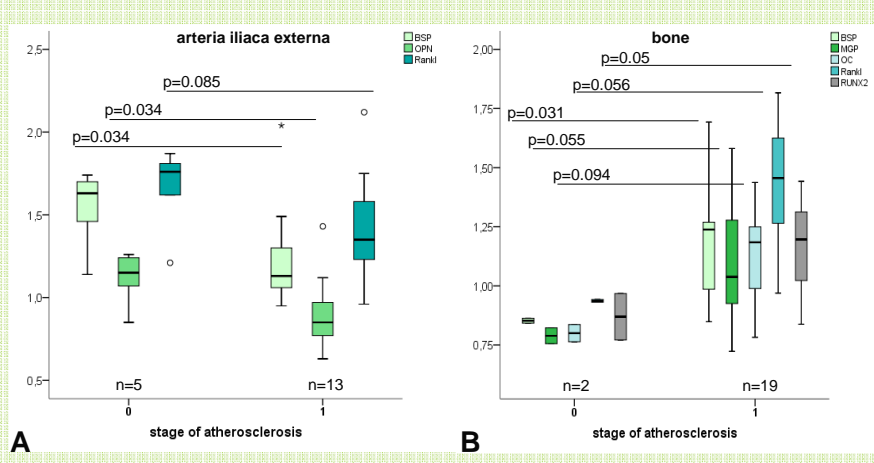

Fig. 3: Changes in the expression of CR (normalised $C p$ values) in 2 stages of atherosclerosis in bone $(A)$ and arteria iliaca (B). Patients were subgrouped in 2 stages of atherosclerosis: Stage $0=$ no changes and stage $1=$ changes in the vessel wall. A: In arteria iliaca externa, $\mathrm{Cp}$ values of BSP-II, MGP and Rankl decreased in the presence of changes in the vessel wall. B: In bone, Cp values of CRs were increased opposed to decreases in the vessels.

\section{Summary:}

- The expression of calcification regulator genes $(\mathrm{CR})$ changes in three histological stages of atherosclerosis.

- In vessels tissue, the expression of CR increases significantly after the first histological signs of calcification in the vessel wall but does not change significantly in further stages.

- At the same time, the expression of CR decreases in bone starting in the stage of vessel wall thickening.

- The expression of Rankl and RUNX2 increases after induction with PTH in HUVECs, but not in HEK293, HOS and EA.hy926 cells.

\section{Conclusion:}

We were able to demonstrate that changes in the expression of calcification regulators occur in the stage of vessel thickening even prior to vessel wall calcification. Changes in the expression of $\mathrm{CR}$ in the vessels were counterbalanced in bone. 